\title{
A experiência na construção de uma cartilha multimídia sobre tuberculose a partir de competências culturais
}

The experience in construction a multimedia primer on tuberculosis based on cultural skills

La experiencia en la construcción de una cartilla multimedia sobre la tuberculosis basada en habilidades culturales

Isabelle Cristine Cardoso do Rosário ${ }^{1 *}$, Elaine Thayna Trindade Costa ${ }^{1}$, Sara do Socorro da Silva Silva ${ }^{1}$, Yasmin Beatriz Reis da Silva Lima ${ }^{1}$, Amanda Rodrigues Pantoja ${ }^{1}$, Myriam Fifame Raphaela Ahlonsou ${ }^{1}$, Nádile Juliane Costa de Castro.

\section{RESUMO}

Objetivo: Descrever a construção de uma cartilha multimídia sobre tuberculose a partir de competências culturais. Relato de experiência: Relata a experiência da construção de uma cartilha multimídia sobre tuberculose baseada na cultura amazônica, por alunas do curso de enfermagem, durante a Atividade Curricular de Atenção Integral à Saúde do Adulto e Idoso, no período do Ensino Remoto Emergencial de uma instituição de ensino superior da Região Norte do país. Essa produção ocorreu entre o final do mês de maio e início do mês de junho de 2021 de forma online, tendo como público alvo os adultos jovens amazônidas dessa região. A elaboração do produto deu-se por meio de três etapas: A pesquisa do tema para fins de roteiro e produção; Construção de um resumo expandido e avaliação da cartilha e resumo pelas docentes. Considerações finais: Foi observado a relevância da criação de materiais educativos por meio de novas tecnologias e a partir de apontamentos que considerem as peculiaridades regionais do público alvo.

Palavras-chave: Autocuidado, Tuberculose, Amazônia, Tecnologias, Saúde da população rural.

\section{ABSTRACT}

Objective: To describe the construction of a multimedia booklet on tuberculosis based on cultural competences. Experience report: Reports the experience of the construction of a multimedia booklet on tuberculosis based on amazon culture, by nursing students, during the Curricular Activity of Integral Care for Adult and Elderly Health, during the period of Emergency Remote Education of a higher education institution in the Northern Region of the country. This production occurred between the end of May and the beginning of June 2021 online, targeting young Amazonian adults from this region. The preparation of the product took place through three stages: The research of the theme for script and production purposes; Construction of an expanded abstract and evaluation of the booklet and abstract by the teachers. Final considerations: It was observed the relevance of the creation of educational materials through new technologies and from notes that consider the regional peculiarities of the target audience.

Keywords: Self-care, Tuberculosis, Amazon, Technologies, Rural population health.

\section{RESUMEN}

Objetivo: Describir la construcción de un folleto multimedia sobre tuberculosis basado en competencias culturales. Informe de experiencia: Relata la experiencia de la construcción de un folleto multimedia sobre tuberculosis basado en la cultura amazónica, por parte de estudiantes de enfermería, durante la Actividad Curricular de Atención Integral a la Salud de Adultos y Ancianos, durante el periodo de Educación Remota de Emergencia de una institución de educación superior en la Región Norte del país. Esta producción ocurrió entre finales de mayo y principios de junio de 2021 en línea, dirigida a jóvenes adultos amazónicos de esta región. La preparación del producto se llevó a cabo a través de tres etapas: La investigación del tema con fines de guión y producción; Construcción de un resumen ampliado y evaluación del folleto y resumen por

${ }^{1}$ Universidade Federal do Pará (UFPA), Belém - PA. *E-mail: cisabelle320@gmail.com 
parte de los profesores. Consideraciones finales: Consideraciones finales: Se observó la relevancia de la creación de materiales educativos a través de las nuevas tecnologías y se basó en notas que consideran las peculiaridades regionales del público objetivo.

Palabras clave: Autocuidado, Tuberculosis, Amazonía, Tecnologías, Salud de la población rural.

\section{INTRODUÇÃO}

A tuberculose (TB) é uma doença infecto-contagiosa, causada pela bactéria Mycobacterium tuberculosis, a qual afeta prioritariamente os pulmões, embora acometa outros órgãos e/ou sistemas. Ela está entre as dez doenças que mais causam óbitos no mundo. A transmissão ocorre de uma pessoa que se encontra com tuberculose ativa e por pessoas suscetíveis que inalam esses aerossóis (FIGUEIREDO JÚNIOR AM, et al., 2021).

O tratamento da TB é gratuito, disponível no Sistema Único de Saúde (SUS), promovendo cura e/ou redução da transmissão. Além disso, o SUS oferta a prevenção da tuberculose por meio da vacina BCG (Bacilo Calmette-Guérin), a qual protege contra as formas mais graves da doença, assim como o tratamento da Infecção Latente da Tuberculose (ILTB), que evita o desenvolvimento da TB ativa (MINISTÉRIO DA SAÚDE, 2021a).

Tal enfermidade continua sendo um grande problema de saúde pública no Brasil, sendo seu controle prioritário pela Organização Mundial de Saúde (OMS). No ano de 2020, o país registrou 66.819 casos novos de TB tendo uma incidência de 31,6 casos por 100 mil habitantes e quando se analisa o coeficiente de incidência dos anos de 2018 e 2019, nota-se que esses anos tiveram cerca de 37,2 e 37,4 casos, respectivamente. Com isso, observa-se que no ano de 2020, momento da pandemia pela Covid-19, houve uma queda acentuada da incidência de casos, comparada aos anos anteriores (MINISTÉRIO DA SAÚDE, 2021b).

Nota-se que entre as pessoas mais vulneráveis a sofrerem com o adoecimento por tuberculose, devido à dificuldade de acesso ao serviço ou às informações. Portanto, infere-se que a tuberculose possui determinantes sociais que corroboram para a manifestação da doença (ZORZI SS, et al., 2019, ALVES KKAF, et al., 2020). Outrossim, populações em situação de vulnerabilidade possuem alto risco de adoecimento por TB, visto que há a falta de acesso integral à saúde, por mais que este seja assegurado pela Política Nacional de Saúde Integral das Populações do Campo e da Floresta (PNSIPCF). Há apontamentos sobre isto, é respeitar as diversidades e especificidades sociais, culturais e biológicas (MINISTÉRIO DA SAÚDE, 2013).

Dessa forma, tendo em vista as circunstâncias sociais de comunidades amazônicas, a utilização de tecnologias educativas em saúde é indubitável para uma eficiente educação em saúde. Essas ferramentas contribuem para a propagação do conhecimento técnico, facilitando o cotidiano. As tecnologias são criadas para auxiliar o usuário perante suas necessidades, a fim de atribuir-lhe mais autonomia (MELO CS, et al., 2020). São um importante meio de promoção da saúde, de criação de hábitos saudáveis e estimulação do autocuidado (MORAIS TMDM, et al., 2017).

Neste sentido, a produção de tecnologias educativas, como cartilhas, tem sido apontada como ferramenta capaz de repassar informações efetivamente, servindo como guia de orientações para casos de dúvidas e auxiliando nas tomadas de decisões do cotidiano. Elas são consideradas um mediador para promover saúde, possibilitando, durante a prática educativa, que o conhecimento seja compartilhado (MOREIRA TMM, 2018).

Para ilustrar melhor as necessidades inerentes ao cenário, buscou-se apreender evidências sobre as competências culturais que devem ser desenvolvidas durante o percurso de construção da cartilha, dentre elas está a habilidade de realizar um cuidado adequado, compreensivo e respeitoso, de forma conciliável com as crenças e práticas culturais de saúde do usuário, e na língua de sua predileção. Além de respeitar e reconhecer a identidade cultural de cada povo, atendendo às suas particularidades, expectativas e direitos (GOUVEIA EAH, et al., 2019).

Diante disso, este trabalho tem como objetivo relatar a experiência da construção de uma cartilha multimídia para a população amazônica, com a finalidade de ajudar a comunidade a identificar os sinais e sintomas da tuberculose e quando procurar atendimento de saúde a partir de competências culturais. 


\section{RELATO DE EXPERIÊNCIA}

O estudo é descritivo do tipo relato de experiência, vivenciado por alunas do $3^{\circ}$ semestre da Faculdade de Enfermagem, a partir da construção de uma cartilha multimídia sobre a tuberculose e construída por meio de referências culturais amazônicas. Foi executada durante Atividade Curricular de Atenção Integral à Saúde do Adulto e Idoso (AISAI), no período do Ensino Remoto Emergencial de uma universidade da Região Norte, com a orientação da docente que ministrava a atividade. A produção deste material teve participação das discentes e da docente da AISAI.

Para elaborar o relato de experiência, utilizou-se a criação da cartilha multimídia que foi produzida no mês de maio e junho de 2021. Tal ferramenta em saúde foi desenvolvida com o intuito de instruir a população da amazônia e afins sobre tópicos essenciais para a prevenção da tuberculose.

A construção da tecnologia em saúde ocorreu por meio de etapas, que são: Etapa 1: a pesquisa do tema, reunião para indicação de roteiro de construção, escolha de editor de imagens e criação do canal, construção, edição, revisão e avaliação do designer e conteúdo; Etapa 2: a construção de um resumo expandido, avaliação parcial e indicações de correções e revisão final e finalização, conforme Figura 1 e 2; Etapa 3: avaliação da cartilha e resumo pelas docentes.

Figura 1 - Etapa 1 do processo de construção da tecnologia.

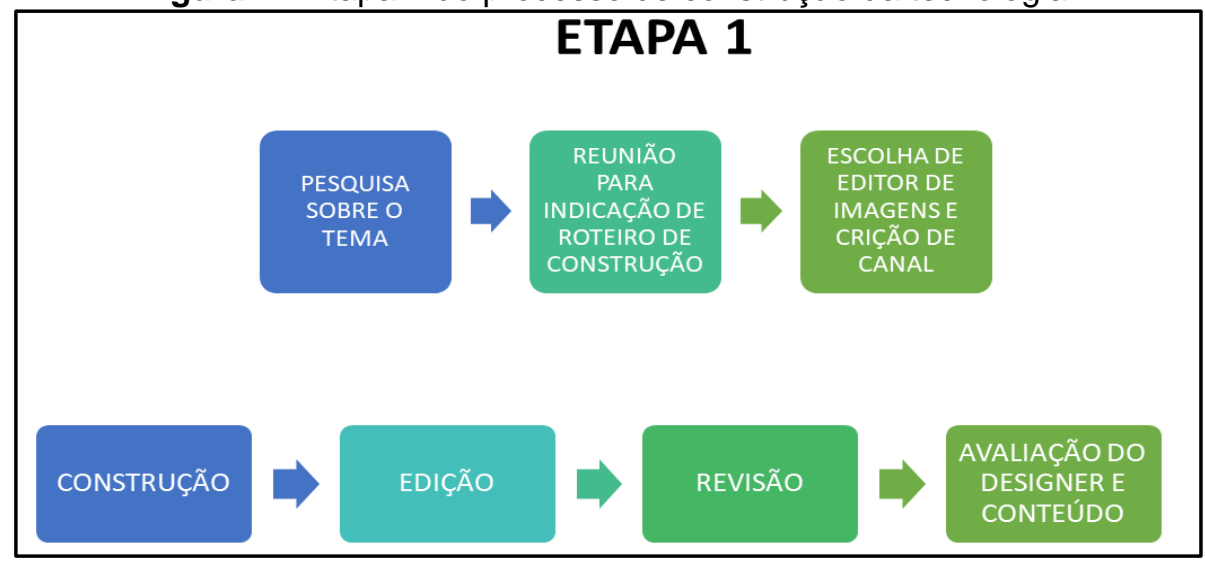

Nota: Imagem produzida no Microsoft Office Power Point@ ${ }^{\circledR}$. Fonte: Rosário ICCD, et al., 2021.

Figura 2 - Etapa 2 do processo de construção da tecnologia.

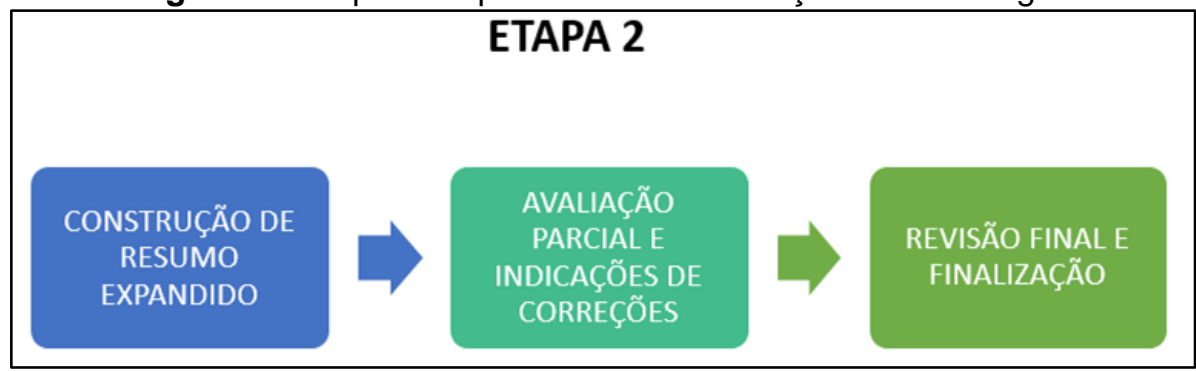

Nota: Imagem produzida no Microsoft Office Power Point@. Fonte: Rosário ICCD, et al., 2021.

A escolha do tema ocorreu por meio de consulta ao site oficial do Ministério da Saúde e nas bases de dados Biblioteca Virtual em Saúde (BVS) e Scientific Electronic Library Online (Scielo) por meio dos seguintes descritores: autocuidado, tuberculose, Amazônia, tecnologias e Saúde da População Rural. Os critérios de inclusão foram as publicações atuais e a partir da combinação dos descritores utilizando o operador boleano "and".

Para a construção do roteiro, foi elencado a sua organização por meio de sete perguntas, que são: O que é tuberculose; Como é a transmissão; Como evitar a transmissão; Quais os sinais e sintomas; Quando procurar atendimento; Como é o tratamento e Como se prevenir. Além disso, incluiu-se a Política Nacional de Saúde Integral dos Povos do Campo e da Floresta (PNSIPCF), a fim de que as populações amazônicas conheçam e entendam sobre a política de saúde que os ampara, definindo deste modo o conteúdo da cartilha. 
Após a seleção do tema a tecnologia foi construída na plataforma de designer gráfico Canva, sendo utilizado para o designer do recurso as cores verde e creme, com a fonte dos textos grande e linhas espaçadas para melhor visualização, combinando com elementos e figuras que o público-alvo se reconheça.

Vale salientar, que houve uma atenção especial, principalmente, para o designer da capa da cartilha, pois buscou-se proporcionar representatividade ao público alvo da região. Para tal, inseriu-se imagem de canoas, do rio e de pessoas dessa comunidade em tarefas do dia a dia, tudo em cor verde remetendo à natureza, inclusive o título do material.

Ademais, houve a criação de um canal de vídeos na plataforma do Youtube, o qual foi criado para a publicação de dois vídeos curtos, com durabilidade de menos de um minuto e de autoria própria, sobre os sinais, sintomas e prevenção, com a finalidade de melhorar a compreensão do público alvo. Estes e outros vídeos de conteúdo extra do Ministério da Saúde, foram expostos em páginas da cartilha por meio de links, representados pelo ícone do Youtube, que direcionam até a plataforma.

A cartilha multimídia possui o título "Olá, Amazônida! Veja o que você precisa saber sobre a tuberculose", sendo constituída por 18 páginas (Figura 3) que são organizadas da seguinte forma: capa, apresentação, nome dos autores, ficha catalográfica, sumário, PNSIPCF, o que é tuberculose, como é a transmissão, como evitar a transmissão, quais são os sinais e sintomas, quando procurar atendimento, como é o tratamento, como se prevenir, referências, links para buscar mais informações e o canal da cartilha, criado no Youtube, quem somos e, por fim, a contracapa. É importante ressaltar que a linguagem verbal e não verbal do material foi pensada para uma leitura rápida e bem instrutiva, com um conteúdo claro e objetivo, a fim de que o assunto abordado possa ser entendido facilmente pelo público alvo.

Figura 3 - Exemplo de algumas páginas da cartilha.

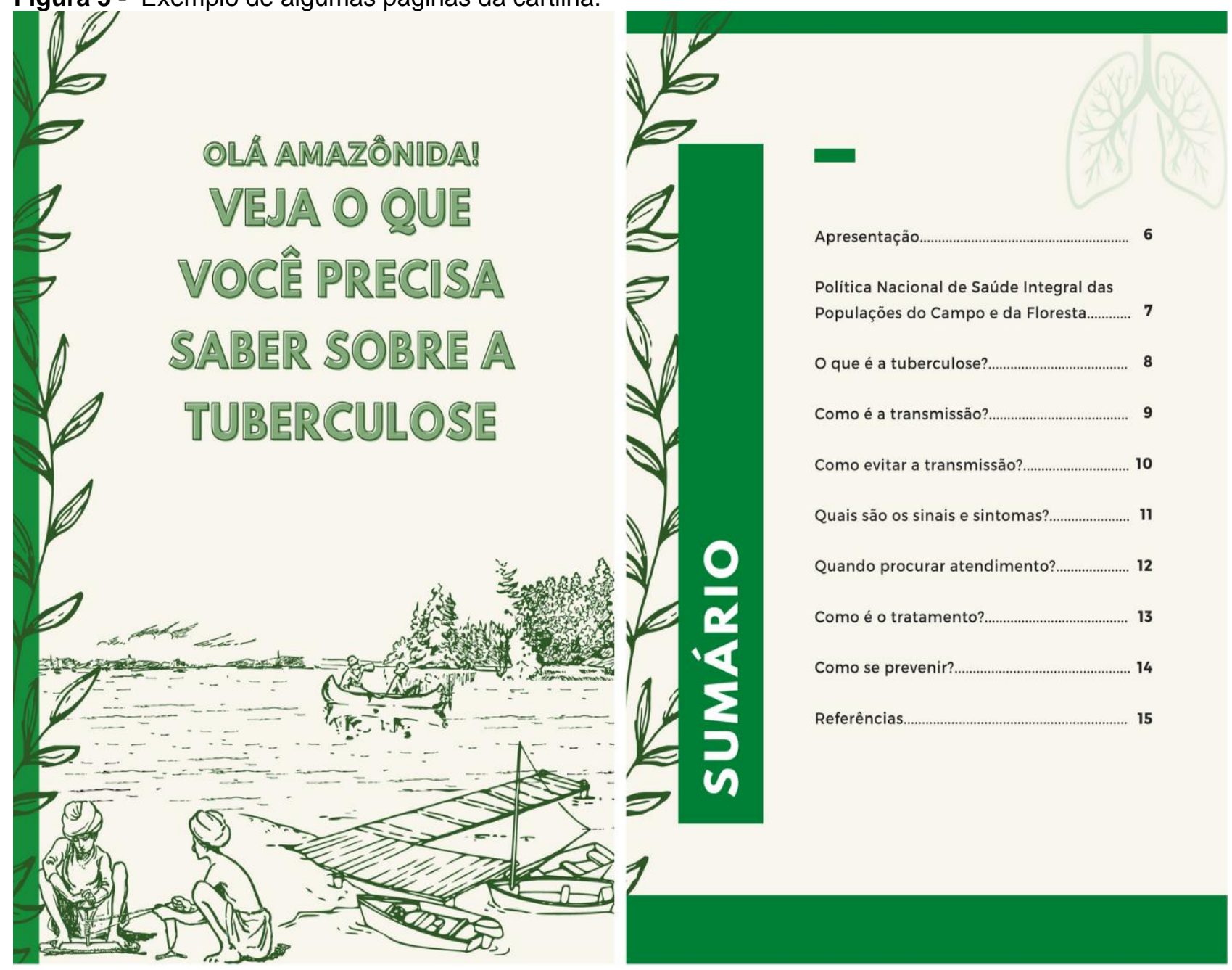




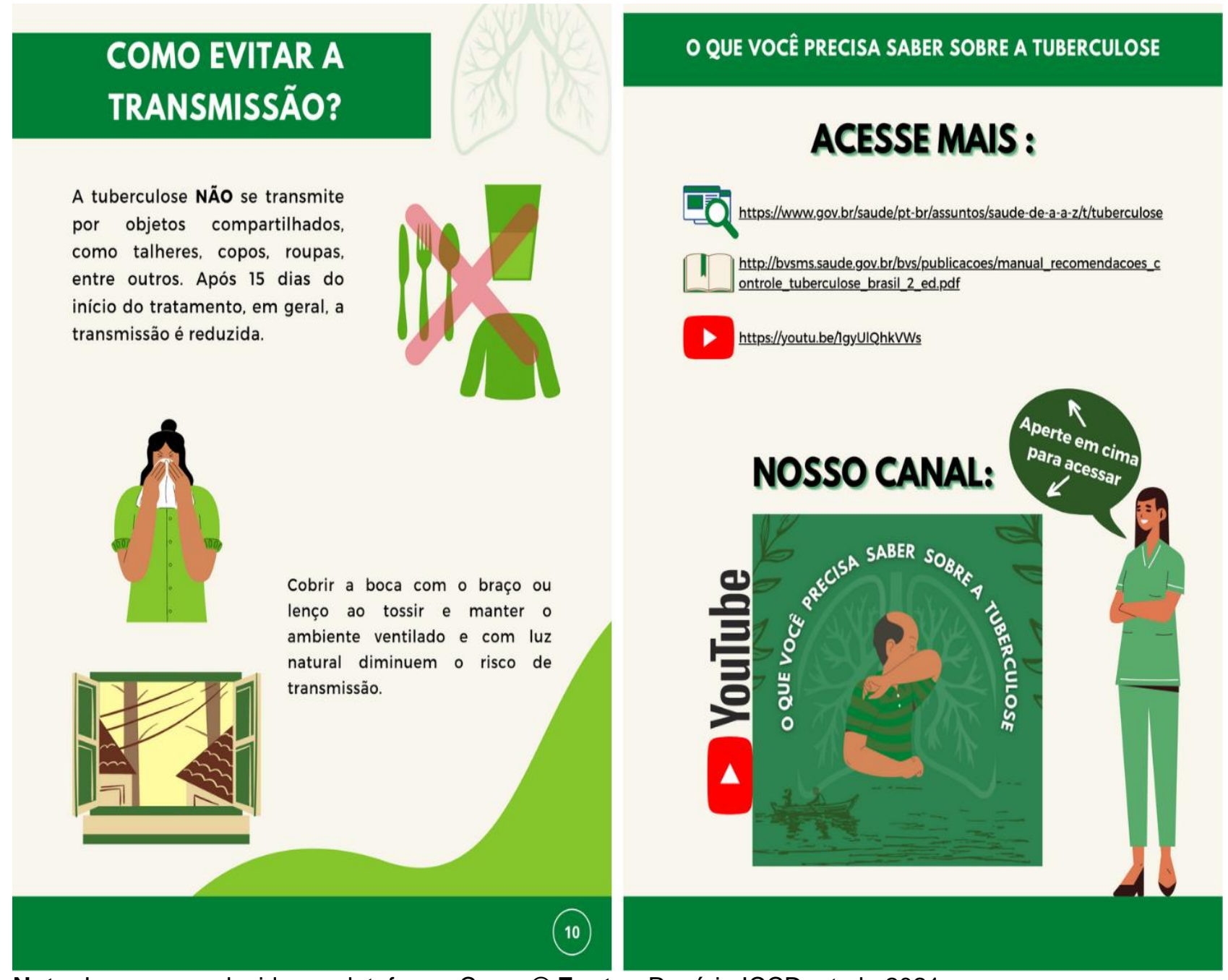

Nota: Imagem produzida na plataforma Canva® Fonte: Rosário ICCD, et al., 2021.

A edição, revisão e avaliação do designer e conteúdo foram avaliados de modo contínuo e crítico, de modo a revelar as potencialidades dos participantes. Importante ressaltar que o processo de valorização da regionalidade foi constantemente assumido na identidade, a fim de evidenciar as questões culturais.

$\mathrm{Na}$ segunda etapa do trabalho, realizou-se a construção de um resumo expandido com finalidade de instituir questões metodológicas. Para isso, foram usadas as etapas de produção da cartilha e a PNSIPCF e a ferramenta em saúde escolhida. A terceira etapa consiste na avaliação da ferramenta em saúde e do resumo, apresentada por meio de exposição.

\section{DISCUSSÃO}

A educação em saúde atua na promoção e prevenção, tendo como objetivo tornar a pessoa autônoma diante da manutenção da sua própria saúde, e deve considerar o contexto social da pessoa ou comunidade a quem irá prestar esse ensino, para que se execute de maneira eficaz. A Enfermagem devido sua proximidade ao cuidado com o usuário de saúde promove orientação mais diretamente. (BENEVIDES JL, et al., 2016; SANTOS FGT, et al., 2021). Para isso, são utilizados meios para o repasse de informações e envolvimento do público alvo, a fim de despertar aptidões para o autocuidado. Nesse âmbito, são usadas tecnologias em saúde com o intuito de alcançar esse objetivo.

Ademais, considera-se que a realização da promoção da saúde na tuberculose deve ser voltada para a discussão de problemas socioeconômicos que envolvem as populações mais atingidas pela doença, como as vulnerabilidades econômica, social, nutricional, habitacional e a falta de acesso à educação de qualidade. 
Essa ação é importante para que toda a informação e conhecimento repassados sejam absorvidos e, dessa forma, a fragilidade relacionada à exposição à tuberculose seja revertida (LARROQUE MM e SANTOS BMO, 2015).

A mediação pelas tecnologias acontece por meio da utilização de instrumentos que integram o saber de enfermagem utilizado pelos profissionais no desenvolvimento diário de sua prática. São associadas à educação permitem o processo de emancipação dos indivíduos envolvidos no cuidar, aplicando-se métodos de cuidados simplificados com a finalidade de tornar sua prática comum, facilitando o autocuidado (NIETSCHE EA, et al., 2012).

Conforme Barros FRB, et al. (2021), as tecnologias em saúde são compostas de métodos, normas, sistemas e dispositivos usados na área da saúde, e são organizadas em leve, leve-dura e dura. (SABINO LMM, 2016). A tecnologia escolhida para a construção é a cartilha, tipo leve-dura, visto que dá-se pela elaboração de conhecimentos operacionalizados nos trabalhos em saúde. Ademais, essa tecnologia serve como reforço das informações orais e acesso rápido em caso de dúvida, por serem elaboradas com uma linguagem fácil e objetiva de acordo com o público alvo (MOREIRA TMM, et al., 2018; SILVA NVN, et al., 2019).

Diante disso, as tecnologias multimídia ajudam nesse processo, pois utilizam ferramentas ligadas a internet, como aplicativos, que proporcionam a conexão com o meio. Nesse sentido, atuam de forma eficiente na área da educação, pois promovem uma comunicação direcionada com cliente do serviço (SANTOS RLN, et al., 2019). $\mathrm{Na}$ atualidade, tornaram-se ainda mais essenciais devido ao aparecimento da pandemia do COVID-19, que teve como uma de suas principais características o isolamento social, sendo assim, as interações sociais tornaram-se de forma virtual (LIRA ALBC, et al., 2020).

Além de escolhas tecnológicas e midiáticas deve-se perceber que é necessário considerar o cenário nos elementos para comunicação da informação. Observou-se que devido a pandemia a educação em saúde teve que ser reformulada para uma ação virtual, considerando o distanciamento social, assim, a construção de uma tecnologia atualmente precisa de recursos que se adeque ao contexto, que seja planejada de forma prática e direcionada (TEIXEIRA E, et al., 2021). É relevante frisar que para a produção desse instrumento, além da circunstância, a aparência, linguagem e o modo como são dispostas as informações devem estar em concordância com o objetivo deste recurso (SOUZA ACC, et al., 2020).

Com isso, a escolha da cartilha levou em consideração, principalmente, o público alvo designado - as comunidades ribeirinhas amazônicas, as quais devido ao seu estilo de vida e localização requerem uma tecnologia que possam ser alcançadas de uma maneira prática e didática. É importante frisar que essa população carece de informações para manutenção da saúde, além de não conhecer as políticas públicas que garantam os direitos ao acesso e qualidade dos serviços (MACHADO TDP, et al., 2020).

Logo, é pertinente destacar a questão cultural na construção da tecnologia, visto que a cultura é dinâmica às diferentes etnias, regiões, hábitos de vida, crenças e a classe socioeconômica de cada grupo social, dessa forma, a cartilha foi produzida pensando nas peculiaridades das comunidades amazônicas. Por conseguinte, conclui-se que foi trabalhado a competência cultural, uma vez que ela é a capacidade de executar uma atenção direcionada ao público alvo, de maneira conforme a identidade do grupo étnico (GOUVEIA EAH, et al., 2019).

Experiências como estas estão sendo apontadas durante a pandemia e por meio do ensino remoto emergencial, visto que, tornou-se necessário buscar diferentes estratégias, com o objetivo de manter e fortalecer a formação dos acadêmicos. Ou seja, a grande produção de diferentes tipos de tecnologias agrega no processo de formação dos estudantes, docentes e profissionais da saúde, além de contribuir consideravelmente no controle e prevenção de doenças (LIRA ALBC, et al., 2020; TEIXEIRA E, et al., 2021).

No decorrer dessa experiência, notou-se a importância da criação de materiais educativos para populações vulneráveis, em especial a ribeirinha. Buscou-se discorrer sobre o que é tuberculose, seus sintomas, tratamentos e epidemiologia, o público alvo e a metodologia utilizada nessa ação. Diante do exposto, esse trabalho visa também contribuir para a disseminação de informação e conhecimento sobre a tuberculose, de forma simples e objetiva, para que seja acessível a essas populações e ainda incentivar a criação de outras tecnologias tão eficientes quanto essa. 


\section{REFERÊNCIAS}

1. ALVES KKAF, et al. Fatores associados à cura e ao abandono do tratamento da tuberculose na população privada de liberdade. Revista Brasileira de Epidemiologia, 2020; 23: e200079.

2. BARROS FRB, et al. Tecnologias desenvolvidas no contexto da saúde da mulher no Brasil: uma revisão integrativa. Revista Cuidarte, 2021; 12(1): e1159.

3. BENEVIDES JL, et al. Construção e validação de tecnologia educativa sobre cuidados com úlcera venosa. Rev Esc Enferm USP, 2016; 50(2): 306-312.

4. FIGUEIREDO JÚNIOR AM, et al. Análise da incidência de tuberculose nos estados da região norte do Brasil. Revista Eletrônica Acervo Científico, 2021; 24: e7041-e7041.

5. GOUVEIA EAH, et al. Competência cultural: uma resposta necessária para superar as barreiras de acesso à saúde para populações minorizadas. Revista Brasileira de Educação Médica, 2020; 43: 82-90.

6. LARROQUE MM, SANTOS BMO. Promoção da saúde e tuberculose. Arquivos de Ciências da Saúde da UNIPAR, $2015 ; 19(3)$.

7. LIRA ALBC, et al. Educação em Enfermagem: desafios e perspectivas em tempos de pandemia COVID-19. Revista Brasileira de Enfermagem, 2020; 73.

8. MACHADO TDP, et al. Percepções de usuários ribeirinhos sobre a atenção à saúde no âmbito da Estratégia Saúde da Família. Rev. Pesqui. (Univ. Fed. Estado Rio J., Online), 2020; 1011-1016.

9. MELO CS, et al. Diga não às síndromes hipertensivas: experiência de produção de tecnologia educacional baseada em evidências. Revista Eletrônica Acervo Saúde, 2020; 58: e4078.

10. MINISTÉRIO DA SAÚDE. Saúde de A a Z: Tuberculose, 2021a. Disponível em: https://www.gov.br/saude/ptbr/assuntos/saude-de-a-a-z/t/tuberculose. Acessado em: 04 de junho de 2021.

11. MINISTÉRIO DA SAÚDE. Secretaria de Vigilância em Saúde. Boletim Epidemiológico: Tuberculose 2021. Número Especial | Mar. 2021. Brasília-DF: Editora MS/CGDI; Brasil, 2021b. 44 p. Disponível em: https://www.gov.br/saude/ptbr/media/pdf/2021/marco/24/boletim-tuberculose-2021_24.03. Acessado em: 04 de junho de 2021.

12. MINISTÉRIO DA SAÚDE. Secretaria de Gestão Estratégica e Participativa, Departamento de Apoio à Gestão Participativa. Política Nacional de Saúde Integral das Populações do Campo e da Floresta. 1. ed.; 1. reimp. Brasília: Editora do Ministério da Saúde, Brasil, 2013. 48 p. Disponível em: https://bvsms.saude.gov.br/bvs/publicacoes/politica_nacional_saude_populacoes_campo.pdf. Acessado em: 04 de junho de 2021.

13. MORAIS TMDM, et al. Cartilha para adultos com síndrome metabólica: Proposta de tecnologia educativa para a promoção da saúde. Anais do Seminário Tecnologias Aplicadas a Educação e Saúde, 2017.

14. MOREIRA TMM, et al. Tecnologias para a promoção e o cuidado em saúde. Fortaleza: Editora da Universidade Estadual do Ceará - $\quad$ Eduece, 2018. $387 \quad$ p. $\quad$ Disponível em: https://www.biosanas.com.br/uploads/outros/artigos_cientificos/59/39ab383257a9da8d6421cc9ada1a1490.pdf. Acessado em: 04 de junho de 2021.

15. NIETSCHE EA, et al. Tecnologias inovadoras do cuidado em enfermagem. Revista de Enfermagem da UFSM, 2012; 2(1): 182-189.

16. SABINO LMM, et al. Uso de tecnologia leve-dura nas práticas de enfermagem: análise de conceito. Aquichan. 2016; 16(2): 230-239.

17. SANTOS FGT, et al. Tecnologia educacional para pessoas com doença renal crônica: construção e validação de conteúdo. Rev. Pesqui. (Univ. Fed. Estado Rio J., Online), 2021; 13: 517-523.

18. SANTOS RLN, et al. O uso de tecnologia multimídia como ferramenta promotora do cuidado de enfermagem em saúde mental. Evento promovido pelo PET/ENFERMAGEM/UECE. 2021.

19. SILVA NVN, et al. Tecnologias em saúde e suas contribuições para a promoção do aleitamento materno: revisão integrativa da literatura. Ciência \& Saúde Coletiva, 2019; 24: 589-602.

20. SOUZA ACC, et al. Desenvolvimento de instrumento para validar aparência de tecnologia educacional em saúde. Revista Brasileira de Enfermagem, 2020; 73.

21. TEIXEIRA E, et al. Tecnologias em tempos de pandemia: aceleração dos processos de produção e publicização. Rev Enferm UFPI, 2021; 10(1).

22. ZORZI SS, et al. Tuberculose em População Indígena Autodeclarada no Estado do Paraná. Rev. bras. ciênc. saúde, $2019 ; 57-64$. 